\title{
Biología Reproductiva de Mytilopsis leucophaeata (Conrad, 1831) (Bivalvia: Dreissenidae) de la Laguna de Tampamachoco, Túxpam-Veracruz
}

\author{
Reproductive Biology of Mytilopsis leucophaeata (Conrad, 1831) (Bivalvia: \\ Dreissenidae) of Tampamachoco Lagoon, Tuxpan-Veracruz
}

"Noemí Pérez-Sarabia; "Esther Uría-Galicia; "Esperanza Ortiz-Ordoñez \& ** Jorge Belmar-Pérez

\begin{abstract}
PÉREZ-SARABIA, N.; URÍA-GALICIA, E.; ORTIZ-ORDOÑEZ, E. \& BELMAR-PÉREZ, J. Biología reproductiva de Mytilopsis leucophaeata (Conrad, 1831) (Bivalvia: Dreissenidae) de la laguna de Tampamachoco, Túxpam-Veracruz. Int. J. Morphol., 30(4):1526$1531,2012$.
\end{abstract}

RESUMEN: Se caracterizó el ciclo reproductivo de Mytilopsis leucophaeata (Conrad, 1831) en organismos colectados mensualmente durante un ciclo anual en la laguna de Tampamachoco, Túxpam, Veracruz, que se ubica en la costa del Golfo de México en la Región Terrestre Prioritaria (RTP-103) de CONABIO considerada sitio RAMSAR 1602 en México. Los resultados histológicos indican que $M$. leucophaeata presenta cuatro etapas gametogénicas: gametogénesis, madurez, desove total para hembras y expulsión parcial para machos. Uno de los principales factores que influyen en el desove sincrónico de gametos tanto en machos como en hembras es la salinidad, en los meses de enero a marzo.

PALABRAS CLAVE: Biología reproductiva; Ciclo reproductivo; Mytilopsis leucophaeata; Gametos; Laguna de Tampamachoco.

\section{INTRODUCCIÓN}

En México existen más de 125 lagunas costeras que en conjunto cubren una superficie aproximada de 12500 $\mathrm{km}^{2}$ (Moreno-Casasola et al., 2006) dentro de éstas, los manglares y humedales revisten un importante hábitat para muchas especies de estuario y marinas. La Laguna de Tampamachoco es el más grande humedal de Túxpam (sitio RAMSAR 1602 y RTP-103 de la Comisión Nacional para el Conocimiento y Uso de la Biodiversidad) (The RAMSAR convention on Wetlands, 2012; Arriaga et al., 2000)

Dentro de la malacofauna de este humedal se encuentra M. leucophaeata (Conrad, 1831), orden Veneroida, también llamado "false mussel" o "falso mejillón obscuro", es un bivalvo dioico, con fecundación externa y se fija a sustratos duros por medio de los filamentos del biso; es eurihalino, en el interior de cada concha posee un diente triangular característico de esta especie.
Los estudios sobre $M$. leucophaeata se han enfocado en los siguientes aspectos: distribución y taxonomía (Reguero \& García-Cubas, 1991) efecto de la temperatura y salinidad sobre la larva de este mejillón (Verween et al., 2007), tolerancia a la salinidad (Wolff, 1969), estudios anatómicos comparativos entre M. leucophaeta y Dreissena polymorpha (Pathy \& Mackie, 1993) y estudios relacionados con los daños que causa en los sistemas de refrigeración (Verween et al., 2002). A la fecha, no existe información sobre la biología reproductiva de $M$. leucophaeata, especie nativa de las costas del Golfo de México, por lo que el presente trabajo tiene como objetivo contribuir al conocimiento de la biología reproductiva de esta especie.

\section{MATERIAL Y MÉTODO}

La Laguna de Tampamachoco se localiza en el estado de Veracruz, en el municipio de Túxpam. Los organismos fueron colectados mensualmente de febrero 2008 hasta

\footnotetext{
* Laboratorio de Histología Animal, Departamento de Morfología, Escuela Nacional de Ciencias Biológicas, Instituto Politécnico Nacional, Ciudad de México, México.

** Departamento de Zoología, Lab. de Ecología, Escuela Nacional de Ciencias Biológicas, Instituto Politécnico Nacional, Ciudad de México, México.
} 
enero de 2009 dentro de la laguna, en la localidad del Tochon $\left(21^{\circ} 19^{\prime} 00.15^{\prime \prime} \mathrm{N}\right.$ y $97^{\circ} 21^{\prime} 04.38^{\prime \prime}$ O) (Fig. 1).

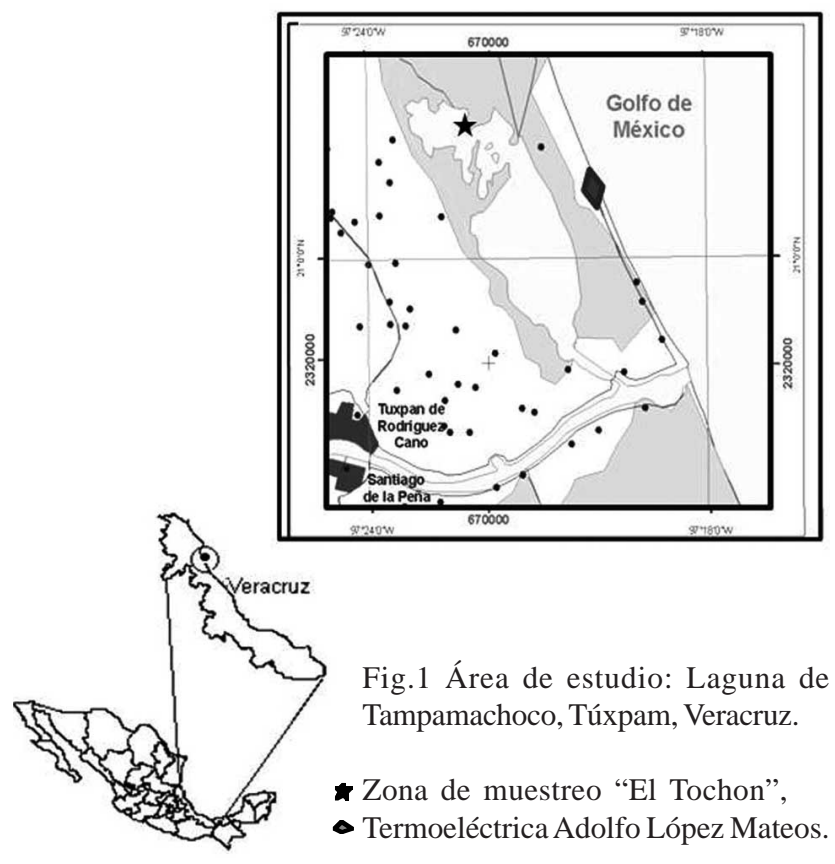

En cada colecta se midieron los valores de temperatura del agua y salinidad, los organismos se obtuvieron de las raíces del mangle rojo (Rhizophora mangle). Estos se desprendieron y fijaron en formaldehido al $10 \%$ con agua de la propia laguna. Transcurrido el tiempo de fijación, se midieron los 600 ejemplares de la colecta anual, para seleccionar 20 ejemplares de la mayor talla en cada mes, se desconcharon y la masa visceral se proceso de acuerdo a la técnica histológica para incluirlas en Paraplast ${ }^{\circledR}$. Se realizaron cortes de $8 \mu \mathrm{m}$ y se tiñeron con la técnica de Hematoxilina-Eosina, las laminillas se analizaron para identificar el sexo, caracterizar las células en desarrollo y las etapas gametogénicas y con base a su frecuencia determinar el ciclo y picos reproductivos que presentan a lo largo del año.

\section{RESULTADOS}

Se encontró que la temperatura del agua más baja fue de $20^{\circ} \mathrm{C}$ en el mes de noviembre y la más alta de $31^{\circ} \mathrm{C}$ en los meses de junio y agosto. La salinidad fue el factor ambiental donde se presentó la mayor variación, ya que el valor más bajo se registró en el mes de octubre siendo de $2,4 \%$ y la mayor salinidad en mayo de $37 \%$. La talla máxima de los organismos fue de $19 \mathrm{~mm}$ y la mínima de $4 \mathrm{~mm}$, seleccionándose de cada mes 20 ejemplares de la mayor talla, para garantizar que estuvieran diferenciados sexualmente, para un total de 240 organismos.

Los resultados histológicos muestran que la gónada de $M$. leucophaeata, esta ubicada tanto en hembras como en machos junto a la glándula digestiva y entre ésta y la región posterior del tubo digestivo en las primeras etapas de maduración; cuando la gónada madura o esta próxima a desovar ocupa la mayor parte de la masa visceral. El tejido conjuntivo ocupa los espacios entre la gónada y el tubo digestivo y disminuye conforme la gónada madura.

La gónada de las hembras se organiza en folículos, éstos contienen a las células gametogénicas y a los ovocitos en diferentes etapas de maduración los cuales ocupan paulatinamente la luz de éstos. La gónada de los machos se organiza en túbulos y en las paredes de estos se desarrollan las células gametogénicas, por lo que los espermatozoides maduros con su flagelo se orientan hacia la luz de los túbulos.

Para la caracterización de las etapas gametogénicas se tomaron en consideración las siguientes características de la gónada de hembras y machos: 1) proporción de folículos o túbulos en relación a la cantidad de tejido conjuntivo que los rodea, 2) grado de desarrollo de las células gametogénicas y 3) proporción entre las células gametogénicas y ovocitos o espermatozoides maduros, con lo cual se definieron cuatro etapas gametogénicas: gametogénesis, madurez, desove total para hembras y expulsión parcial para machos, las cuales se describen a continuación.

\section{Etapas de desarrollo en hembras.}

Gametogénesis. Esta etapa se caracteriza por presentar escasos folículos conteniendo principalmente ovogonias que rodean la pared del folículo formando en ocasiones grupos celulares y escasos ovocitos en desarrollo y pedunculados con núcleo y nucléolo evidente y relación núcleo citoplasma 1:1 unidos al epitelio folicular. El tejido conjuntivo es muy abundante. El porcentaje más alto (100\%) de los organismos examinados en los meses de mayo y junio se encontraron en esta fase (Figs. 2A y 3B).

Madurez. El tamaño de los folículos aumenta y se extiende hacia la glándula digestiva, en esta etapa se encuentran escasas ovogonias y ovocitos en desarrollo predominado los ovocitos maduros libres en la luz de los mismos. Disminuye notablemente la cantidad de tejido conjuntivo, debido al incremento del tamaño de los folículos. El porcentaje más alto (100\%) de los organismos examinados en los meses de julio a septiembre y noviembre se encontraron en esta fase (Figs. 2B y 3B). 

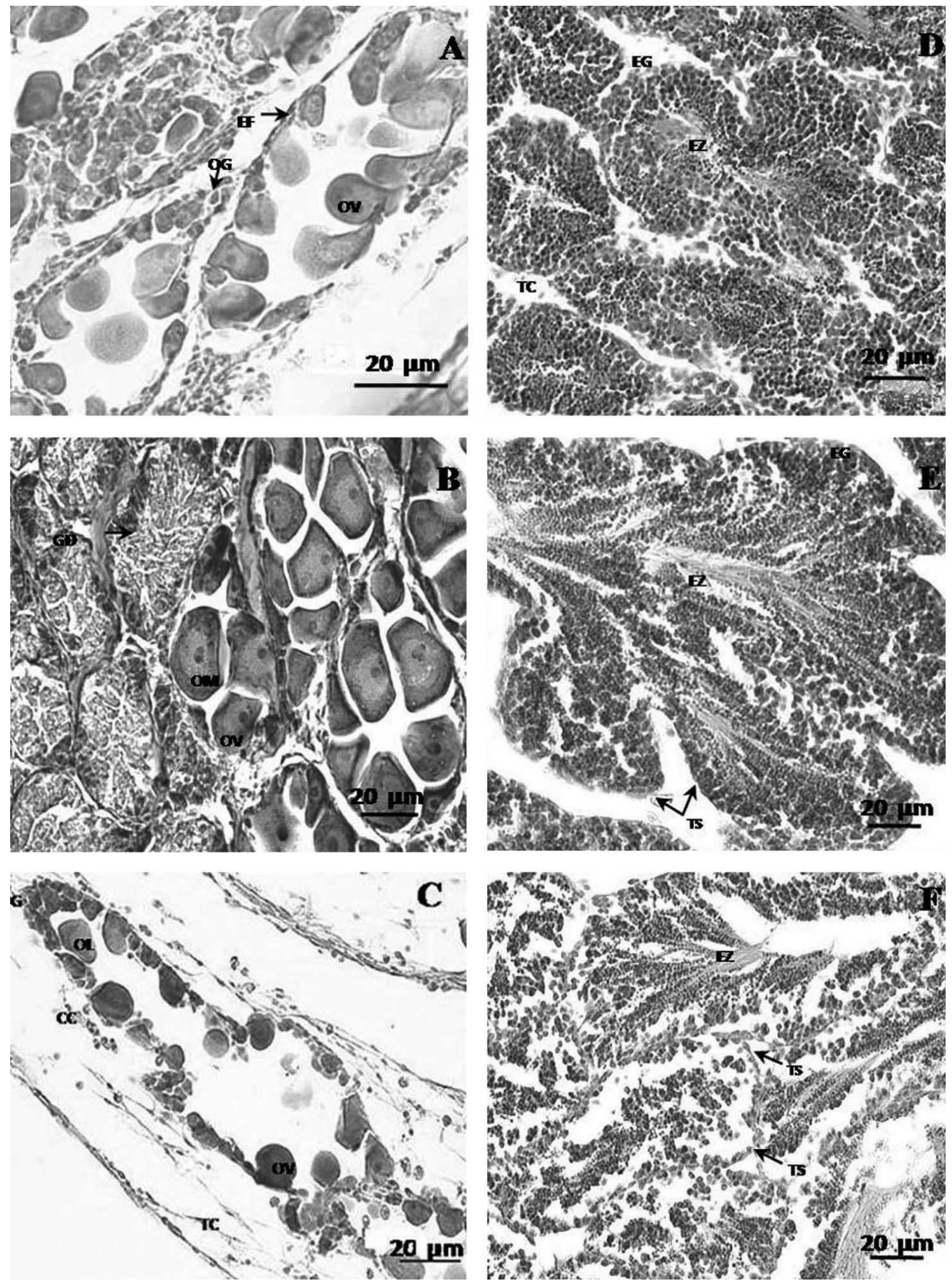

Fig. 2A Gónada femenina de Mytilopsis leucophaeata, Etapa de gametogénesis. 2B Etapa de madurez y 2C Etapa de desove total. (OG) Ovogonias, (OV) Ovocitos en diferentes etapas de desarrollo, (OM) Ovocitos maduros, (TC) Tejido conjuntivo. (GD) Glándula digestiva, (EF) Epitelio folicular, (OL) Ovocito libre, (CC) Células cafés. Téc. Hematoxilina - Eosina. Fig. 2D Gónada masculina de Mytilopsis leucophaeata, Etapa de gametogénesis. 2E Etapa de madurez y 2F Etapa de expulsión parcial. (EG) Espermatogonias, (EZ) Espermatozoides, (TS) Túbulo seminífero. Téc. Hematoxilina - Eosina. 
Desove total. Las paredes de los folículos se fusionan dando origen a folículos de gran tamaño conteniendo escasas ovogonias, ovocitos en desarrollo y maduros libres debido a que la mayoría han sido desovados. Se observan células cafés cercanas a los folículos y el tejido conjuntivo comienza a aumentar notablemente. El porcentaje más alto $(100 \%)$ de los organismos examinados en los meses de enero a abril y octubre se encontraron en esta fase (Figs. 2C y 3B).

\section{Etapas de desarrollo en machos}

Gametogénesis. En la pared de los túbulos se presentan varias capas de células gametogénicas predominando las espermatogonias y en menor cantidad se observan espermatozoides maduros en la luz de los mismos. El tejido conjuntivo es abundante. El porcentaje más alto (100\%) de los organismos examinados en los meses de abril a julio se encontraron en esta fase (Figs. 2D y 3A).
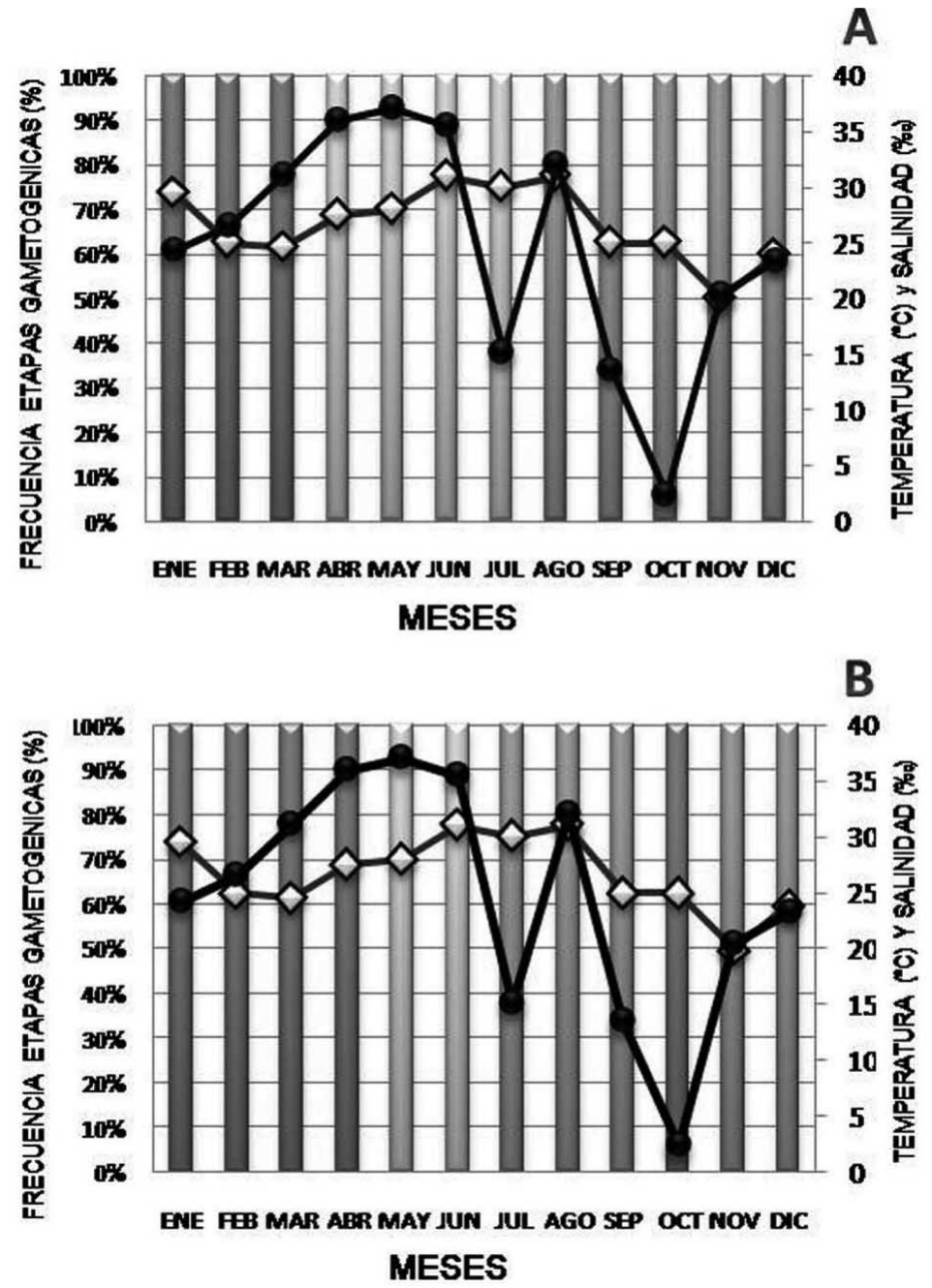

Fig. 3 Frecuencia de etapas gametogénicas de Mytilopsis leucophaeata a lo largo del año A) Machos. B) Hembras.

Expulsión parcial y desove $\square \quad$ Madurez

Gametogénesis $\square \quad$ Salinidad $\bigcirc \quad$ Temperatura del agua $\diamond$
Madurez. Las espermatogonias disminuyen, los espermatozoides maduros son muy abundantes y ocupan la mayor parte de la luz del túbulo. Es evidente la disminución del tejido conjuntivo. El porcentaje más alto (100\%) de los organismos examinados en los meses de agosto a diciembre se encontraron en esta fase (Figs. 2E y 3A).

Expulsión parcial. Las paredes de los túbulos se fusionan en algunos casos y se observan en la pared de los mismos escasas espermatogonias, predominando los espermatozoides maduros libres o empaquetados en la luz. Hay un incremento en la cantidad de tejido conjuntivo. El porcentaje más alto $(100 \%)$ de los organismos examinados en los meses de enero a marzo se encontraron en esta fase (Figs. 2F y $3 \mathrm{~A}$ ).

\section{DISCUSIÓN}

Mytilopsis leucophaeata es un bivalvo dioico, con fecundación externa, la reproducción es cíclica y estos ciclos reproductivos pueden ser anuales, semianuales o continuos, dependiendo de las especies y su localización (Martínez, 2002). En el caso de $M$. leucophaeata se encontró que su ciclo reproductivo es continuo, coincidiendo con lo reportado por Bückle Ramírez \& Díaz (1996) para el ciclo gonádico de Pteria sterna y Arreola Hernández (1997) para Dosinia ponderosa.

Dentro del ciclo reproductivo de Mytilopsis leucophaeta se encontraron las siguientes etapas gametogénicas: gametogénesis, madurez, desove total para las hembras y expulsión parcial para los machos, los resultados encontrados son similares a aquellos obtenidos para otros organismos como Chione californiensis (García-Domínguez et al., 1993), Megapitara aurantiaca (Baqueiro \& Stuardo, 1977) e Isognomon alatus (García-Pacheco, 2004).

Especies tropicales como los Venéridos Anomalocardia brasiliana (Grotta \& Lunetta, 1980), Meretrix meretrix, M. casta y Katelysia pina (Jayaval, 1986), tienen periodos de reproducción extendidos o largos al igual que lo encontrado en M. leucophaeta que presenta un ciclo reproductivo largo con un pico de desove sincrónico en los meses de enero a marzo. 
Andrade (1997) y Velez (1991) mencionan que los cambios drásticos en la salinidad pueden inducir desoves masivos y presentarse picos de desove cuando hay variaciones marcadas en la salinidad relacionadas con la época de lluvias, lo cual coincide con lo observado en M. leucophaeata en el mes de octubre, donde hubo una precipitación pluvial atípica favoreciendo el desove de las hembras ocasionando un pico reproductivo evidente, solo para las hembras lo cual no garantizaría la fecundación por que los machos se encuentran en etapa de madurez.

Para M. leucophaeata se encontró que el factor que influye principalmente es la salinidad y no la temperatura, como se ha observado en otros Veneroideos como Venerupis japonica (Holland \& Chew, 1974), Ameghinomya antiqua (Verdinelli \& Schuldt, 1976), Venus antiqua (Lozada \& Bustos, 1984). Cuevas-Guevara \& Martínez-Guerrero (1979) mencionan que la actividad reproductora puede estar determinada también por una interacción entre factores exógenos como la latitud, alimentación, temperatura y salinidad lo cual puede influir también sobre la actividad reproductora de $M$. leucophaeata.

Por otra parte la malacofauna de la Laguna de Tampamachoco, Túxpam de Rodríguez Cano, Veracruz de Ignacio de la Llave (Instituto Nacional de Estadística y Geografía, 2012) con el transcurso de los años ha sido afectada debido a los asentamientos urbanos, agropecuarios e industriales (Basañez Muñoz et al., 2008), también la industria pesquera por que emplea lanchas con motor fuera de borda (Botello \& Calva, 1998), la termoeléctrica Adolfo López Mateos de la Comisión Federal de Electricidad, contribuye también a la contaminación por metales pesados (Díaz Flores, 1994). En México la NOM-001-ECOL-1996 establece los límites permisibles en materia de metales pesados en las descargas de aguas residuales y bienes nacionales (ríos, estuarios, humedales y suelo de humedales). Sin embargo los metales pesados determinados en agua, sedimento y biomarcadores de daño temprano en la lebrancha (Mugil curema) superan los límites establecidos por la NOM-001ECOL-1996; por lo cual la laguna presenta un alto grado de contaminación, pudiendo afectar por lo tanto a los ciclos gametogénicos de la malacofauna que habita en esta zona, influyendo en la duración e incluso el número de ciclos (Bautista, 1989), lo cual puede estar repercutiendo actualmente en el ciclo reproductivo de Mytilopsis leucophaeata por lo que estudios posteriores demostrarían si la población se mantiene con las condiciones que se encuentran actualmente o aumenta debido a su capacidad de adaptación, siendo favorecida por el desplazamiento de otras especies.

AGRADECIMIENTOS. Este trabajo fue apoyado por el Instituto Politécnico Nacional. COFAA. Forma parte del proyecto SIP 20091612 "Biología Reproductiva de Mytilopsis leucophaeata (Corad, 1831) (Bivalvia, Dreissenacea, Dreissenidae) de la Laguna de Tampamachoco, Tuxpám Veracruz.

PÉREZ-SARABIA, N.; URÍA-GALICIA, E.; ORTIZ-ORDOÑEZ, E. \& BELMAR-PÉREZ, J. Reproductive biology of Mytilopsis leucophaeata (Conrad, 1831) (Bivalvia: Dreissenidae) of Tampamachoco lagoon, Túxpam-Veracruz. Int. J. Morphol., 30(4):15201525, 2012.

SUMMARY: The reproductive cycle of Mytilopsis leucophaeata (Conrad, 1831) was characterized in organisms collected monthly during an annual cycle in Tampamachoco lagoon Tuxpam-Veracruz, located on the coast of the Gulf of Mexico in the Terrestre Prioritaria Region (RTP-103) of CONABIO considered a RAMSAR 1602 site in Mexico. Histological results indicated that M. leucophaeata has four gametogenic stages: gamete, maturity, female's total spawning and partial expulsion inmales. One of the main factors that influence the synchronous spawning gametes in both males and females is salinity, in the months of January to March.

KEY WORDS: Reproductive biology; Reproductive cycle; Mytilopsis leucophaeata; Gametes; Tampamachoco Lagoon.

\section{REFERENCIAS}

Andrade, I. Crassostrea rhizophorae (Guilding) and Crassostrea brasiliana (Lamark) in South and Central America. En: Menzel, W. (Ed.). Estuarine and marine bivalve cultura. Boca Raton, Florida, CRC Press, 1997. pp.125-34.

Arreola Hernández, J. F. Aspectos Reproductivos de Dosinia ponderosa, Gray, 1838 (Bivalvia:Veneridae) en Punta Arena, Bahía Concepción B.C.S. Tesis de Maestría, La Paz, CICIMAR-Instituto Politécnico Nacional, 1997. p.82.
Arriaga, L.; Espinoza, J. M.; Aguilar, C.; Martínez, E.; Gómez, L. \& Loa, E. Regiones terrestres prioritarias de México. México, Comisión Nacional para el Conocimiento y uso de la Biodiversidad, 2000.

Baqueiro, E. \& Stuardo, J. Observaciones sobre la biología, ecología y explotación de Megapitara aurantiaca (Sow., 1835), M. squalida (Sow., 1835) y Dosinia ponderosa (Gray, 1838) (Bivalvia: veneridae) de la Bahía de Zihuatanejo e Isla Ixtapa, Gro., México. An. Inst. Cienc. del Mar y Limnol. Univ. Nal. Autón. México, 4(1):161-208, 1977. 
Basañez Muñoz, A. J.; Cruz Lucas, M. A.; Domínguez Barradas, C.; González Gándara, c.; Serrano Solís, A. \& Hernández Azuara, A. Estructura y producción de Conocarpus erectus L. en el Sitio Ramsar "Manglares y Humedales de Tuxpan", Veracruz, México. Revista UDO Agrícola, 8(1):78-87, 2008.

Bautista, C. Tecnología de Moluscos. Madrid, Ed. Mundi-Prensa, 1989. p.166

Botello, A. V. \& Calva, B. L. G. Polycyclic aromatic hydrocarbons in sediments from Pueblo Viejo, Tamiahua and Tampamachoco Lagoons in the Southem Gulf of Mexico. Bull. Environ. Contam. Toxicol., 60(1):96-103, 1998.

Bückle Ramírez, L. F. \& Díaz, A. H. Ciclo gonádico de Pteria sterna (Gould, 1851) (Mollusca, Bivalvia) en Baja California México. Ciencias Marinas, 22(004):450-509, 1996.

Cuevas-Guevara, C. A. \& Martínez-Guerrero, A. Estudio gonádico de Crassostrea corteziensis Hertlein, C. palmula Carpenter y $C$. iridescens Hanley, de San Blas, Nayarit, México (Bivalvia: Ostreidae). Anales del Centro de Ciencias del Mar y Limnología, 6:81-98, 1979.

Díaz Flores, V. Caracterización preliminar de la presencia de metales en las lagunas de Tamiahua, Pueblo Viejo Tampamachoco, Veracruz, México. México D. F., Universidad Autónoma Metropolitana, División de Ciencias Biológicas y de la Salud. Servicio Social, 1994.

García-Domínguez, F.; García-Melgar, G. \& González-Ramírez, P. Ciclo reproductivo de la almeja roñosa Chione californiensis (Broderip, 1835) en Bahía Magdalena, B.C.S., Mexico. Ciencias Marinas, 19:528, 1993.

García-Pacheco, R. Evolución Gonádica a Nivel Histológico de la almeja Isognomon alatus (Gmelin, 1791) (Mollusca: Bivalvia) de la laguna La Mancha, Veracruz, México. Tesis de Licenciatura, Iztapalapa, Universidad Autónoma Metropolitana, 2004. p.33.

Grotta, M. \& Lunetta, J. E. Ciclo sexual de Anomalocardia brasiliana (Gmelin, 1791) do litoral de Estado de Paraiba. Rev. Nordestina Biol., 3(1):5-55, 1980.

Holland, D. A. \& Chew, K. K. Reproductive cycle of the Manila clam (Venerupis japonica) from. Hood Canal, Washington. Proc. Natl. Shellfish Assoc., 64:53-8, 1974.

Instituto Nacional de Estadística y Geografía (INEGI). Veracruz de Ignacio de la Llave, Municipio (Túxpam de Rodríguez Cano) y Cabeceras municipales. 2012. Disponible en: http:// mapserver.inegi.gob.mx/geografia/espanol/datosgeogra/basicos/estados/ver_geo.cfm

Jayabal, R. Reproductive cycles of some bivalves from velar Estuary, East coast of India. Indian J. Mar. Sci., 15(1):59-60, 1986.

Lozada, E. \& Bustos, H. Madurez sexual y fecundidad de venus antiqua antiqua King y Broderip 1835 en la Bahía Ancud (Mollusca: Bivalvia: Veneridae). Rev. Biol. Mar., 20(2):91-112, 1984.

Martínez, D. Estudio de los solénidos. Solen marginatus (Pennánnt, 1777) y Ensissiliqua (Linné, 1758), de los bancos naturales de la Ría de
Ortigueira y Ría del Barquero: ciclo gametogénico, composición bioquímica y cultivo larvario. Tesis doctoral. Santiago de Compostela, Universidad de Santiago de Compostela, 2002. p.240.

Moreno-Casasola, P.; Peresbarbosa, R. E. \& Travieso-Bello, A. C. Estrategias para el manejo costero integral: el enfoque municipal. México, Instituto de Ecología, A.C. Gobierno del Estado de Veracruz, 2006.pp.21-50.

Pathy, D. A. \& Mackie, G. L. Comparative shell morphology of Dreissena polymorpha, Mytilopsis leucophaeata, and the "quagga" mussel (Bivalvia: Dreissenidae) in North America. Can. J. Zool. 71(5):1012-23, 1993

Reguero, M. \& García-Cubas, A. Moluscos de la laguna Tampamachoco, Veracruz, México: Sistemática y ecología. An. Inst. Cienc. del Mar y Limnol. Univ. Nal. Autón. México, 18(2):289-328, 1991.

The RAMSAR convention on Wetlands. 2012. Disponible en: http:// www.ramsar.org/cda/es/ramsarhome/main/ramsar/ 1\%5E7715_4000_2

Velez, A. Biology and culture of the Caribbean mangrove oyster, Crassostrea rhizophorae, Guilding, in the Caribbean and South América. En: Menzel, W. (Ed.): Estuarne and marine bivalve culture. Boca Raton, Florida, CRC Press, 1991. pp.117-24.

Verdinelli, M. A. \& Schuldt. M. Consideraciones preliminares sobre aspectos de dinámica poblacional y reproducción de la almeja rayada (Ameghinomya antiqua King, Chionidae) en Punta Loma, Golfo Nuevo, Chubut. Rev. Mus. La Plata, Secc. Zool., 12:183-202, 1976.

Verween, A.; Degraer, S. \& Vincx, M. Reducing the economic impact of an invasive bivalve, Mytilopsis leucophaeata, in the harbour of Antwerp Bull. Kon. Belg. Inst. Natuurwet. Biologie, 72(Suppl.):71, 2002.

Verween. A.; Vincx, M. \& Degrader, S. The effect of temperature and salinity on the survival of Mytilopsis leucophaeata larvae (Mollusca, Bivalvia): The search for environmental limits. J. Exp. Mar. Biol. Ecol., 348(1-2):111-20, 2007.

Wolff, T. The Mollusca of the estuarine region of the rivers Rhine, Meuse and Scheldte in relation to the hydrography of the area. II. The Dreissenidae. Basteria, 33(5-6):93-103, 1969.

Dirección para Correspondencia:

Noemí Pérez-Sarabia

Laboratorio de Histología Animal

Departamento de Morfología

Escuela Nacional de Ciencias Biológicas

Instituto Politécnico Nacional

Prolongación de Carpio esq. Plan de Ayala S/N

Col. Casco de Sto. Tomás. C.P. 11340

MÉXICO

Email: nazomi1286@hotmail.com

Recibido : 27-01-2012

Aceptado: 06-06-2012 